\title{
Accurate and continuous ultrasonography evaluation of small diameter vascular prostheses in vivo
}

\author{
JING SHI $^{1 *}$, JIALING ZHANG ${ }^{2 *}$, MENG YIN $^{2}$, QIAN WANG ${ }^{1}$ and JUN DU ${ }^{1}$ \\ ${ }^{1}$ Imaging Diagnosis Center; ${ }^{2}$ Department of Cardiothoracic Surgery, Shanghai Children's Medical Center, \\ Shanghai Jiao Tong University School of Medicine, Shanghai 200127, P.R. China
}

Received August 8, 2017; Accepted January 5, 2018

DOI: $10.3892 /$ etm.2018.5895

\begin{abstract}
There is a large clinical requirement for novel vascular grafts; however, the development of novel vascular grafts has not been extremely successful to date. The most successful method for the continuous evaluation of vascular grafts in vivo remains unclear. Therefore, an optimal successive, non-invasive imaging modality is necessary for the study of vascular transplantation. In the present study, a common rabbit model of carotid artery defect was utilized. The patency and hemodynamic characteristics of implanted grafts was examined following surgery by color Doppler ultrasound in three modes, including B-mode, color flow map and pulse-Doppler examination. The results revealed that ultrasound had sufficient spatial resolution to generate clear images of the carotid artery of rabbits with or without the implanted grafts. Color Doppler ultrasound may be applied to evaluate and differentiate the patent, stenosis and occlusion of carotid arteries in rabbits with different vascular grafts implanted. Furthermore, color Doppler ultrasound is an optimal imaging modality for continuous evaluation in vivo. It is also possible for some quantitative analyses to be performed, including measuring the diameter of vascular lumens and the flow velocity of the region of interest. The present study suggests vascular ultrasound as the optimum choice for continuous surveillance of vascular prostheses in vivo, which may provide valuable information about the grafts in order to greatly shorten the experimental period.
\end{abstract}

Correspondence to: Dr Jun Du or Dr Qian Wang, Imaging Diagnosis Center, Shanghai Children's Medical Center, Shanghai Jiao Tong University School of Medicine, 1678 Dong Fang Road, Shanghai 200127, P.R. China

E-mail: dujunscmc@163.com

E-mail: wangqian@scmc.com.cn

*Contributed equally

Key words: small-diameter blood vessels, non-invasive monitoring, ultrasound, in vivo

\section{Introduction}

Synthetic vascular grafts are widely required in a variety of clinical diseases, including peripheral arterial disease, coronary artery disease, congenital heart disease and arteriovenous access (1). Animal models may be used to simulate the human anatomy, physiology and pathological processes, and are widely applied in tissue engineering experiments prior to clinical translation. To enable long-term vessel substitution studies of animal models in vivo, a suitable succession imaging monitor modality is essential.

Polymer grafts have been used and studied widely since the 1950s $(2,3)$; however, they have demonstrated limited success in the area of small-diameter vessels $(<6 \mathrm{~mm})$ owing to the occurrence of acute and sub-acute thrombosis (4). Hitherto, modification of the small-diameter grafts for long-term patency is the primary objective of several tissue engineering groups (5-7); however, in vivo observation is challenging due to the small diameter. Thus, the present study focused on the study of an in vivo succession monitor imaging modality of animal models implanted with small-diameter grafts.

The evaluation of the patency of vessels and blood flow characteristics following vascular graft implantation surgery in animals is performed using angiography, the gold standard (8). However, it is an invasive monitoring method, which may lead to surgical complications, including hyperanesthesia, bleeding, lameness and possibly early humane euthanasia related to the complications (9-11). Therefore, an alternative non-invasive technique that provides rapid, safe, reproducible and reliable results is essential to reduce animal sacrifices and to benefit long-term experimental observations $(12,13)$.

Several non-invasive imaging modalities exist, including multidetector computed tomography angiography (MDCTA) (14), magnetic resonance angiography (MRA) (15) and vascular Doppler ultrasonography (16). Doppler ultrasound is a frequently applied method used to assess the rates of stenosis of main arteries in humans (14). It includes three modalities: B-mode (grayscale), color Doppler evaluation and velocity measurements. B-mode allows an optimal two-dimensional (2D) anatomic assessment, measurements of diameter, and the observation of lumen acoustic transmission and surrounding soft tissues. Color Doppler allows the visualization of flow abnormalities, including color filling defect of the grafts and turbulence related to the presence 
of stenosis. Finally, the spectral Doppler measures the blood flow velocity and is the cornerstone of the functional evaluation of grafts (17). The blood flow velocity is the quantitative parameter for grading the patency of synthetic vascular grafts and may provide valuable information on critical parameters, including i) the morphology and composition of vascular grafts, ii) the patency of stent and graft materials and iii) the localization and remodeling of the implant materials over a particular time period (18). The present research group speculated that ultrasound would be an excellent screening method in animal models due to its non-invasiveness, availability and successful applications in humans (19).

Currently, the research of the present group is focused on exploring small-diameter polymer vascular grafts in vivo. In a previous study by the present research group, a novel orthogonally functionalizable poly(ester urethane)urea with disulfide and amino groups (PUSN) was developed for surface covalent cofunctionalization, which may be electrospun into fibrous grafts. The scaffolds were successfully modified by heparin (Hep; PUSN-Hep) and Hep/EPC recruiting peptide (TPS) peptide (PUSN-Hep/TPS) to increase the anticoagulant properties and effects of blood compatibility and proliferations (20). Thus, the orthogonally functionalized PUSN grafts demonstrated potential for application in small-diameter vascular regeneration (20). However, the translation of such vascular grafts into clinically useful products that are suitable for implantation into patients has been slow (21-23). One of the clinical transformation difficulties involves monitoring the position and performance of vascular prostheses upon implantation (24). Thus, an intravital technique is required for continuous monitoring of the development of vascular prostheses implanted in animal models without animal dissection due to long-term patency. Among all animal models, the carotid artery of the rabbit is a classic model for small-diameter grafts (25).

Therefore, the present study utilized vascular ultrasound to monitor the synthetic vascular graft implanted into the carotid arteries of rabbits every week.

\section{Materials and methods}

Animals. A total of 15 male New Zealand white rabbits ( 8 weeks old, $2.5 \pm 0.6 \mathrm{~kg}$ ) were purchased from Shanghai Chedun Experimental Animal Raising Farm (Shanghai, China) for use in the present in vivo study of three kinds of fibrous vascular grafts [PUSN, PUSN-Hep and PUSN-Hep/TP, which were manufactured by our group as described previously (20); $n=5 /$ group for each graft]. Rabbits were maintained in a controlled environment $\left(24^{\circ} \mathrm{C} ; 45-65 \%\right.$ humidity and were exposed to a 12-h light/dark cycle) and had free access to food and water. The patency rates of all grafts were analyzed using Origin 8.0 (Origin Lab Corp., Northampton, MA, USA). All experimental protocols were approved by the Animal Care and Experiment Committee of Shanghai Jiao Tong University School of Medicine (Shanghai, China).

Surgical procedures. Rabbits were anesthetized with $30 \mathrm{mg} / \mathrm{kg}$ pentobarbital sodium (Merck KGaA, Darmstadt, Germany) by intravenous injection at the edge of the ear, and $100 \mathrm{U} / \mathrm{kg}$ Hep
(Shanghai Pharma No. 1 Biochemical \& Pharmaceutical Co., Ltd., Shanghai, China) was administered by intravenous injection at the edge of the ear for anticoagulation prior to surgery. A length of $\sim 1.0 \mathrm{~cm}$ of the right carotid artery of each rabbit was removed, and the vascular graft with a $2 \mathrm{~mm}$-diameter and 1.2-cm length was sewn in an end-to-end fashion using 9-0 monofilament nylon sutures. Following this, the skin was closed with 3-0 nylon sutures under stringent aseptic conditions. Aspirin (AstraZeneca, Wuxi, China) was administrated daily $(2 \mathrm{mg} / \mathrm{kg})$ by oral administration as an anticoagulant for 1 week.

Ultrasound examinations. Ultrasound examinations of the carotid arteries of the rabbits were performed at the Laboratory at Shanghai Children's Medical Center, Shanghai Jiao Tong University School of Medicine by an experienced sonographer. The patency of the implanted grafts was monitored by a color Doppler ultrasound diagnostic system (GE LOGIQ 9; GE Healthcare, Chicago, IL, USA) 4 and 8 weeks after implantation using an M12L multi high-frequency linear transducer (GE Healthcare, Chicago, IL, USA).

For the examination, the rabbit's head was in the supine position and turned away from the examination side. The right common carotid artery with the implanted graft and its forward and distal carotid vessel region constituted the region of interest, and the left common carotid represented the control.

Firstly, in the grayscale mode, the sound beam was adjusted perpendicular to the wall of the graft, focusing on the acquisition of two parallel echogenic lines corresponding to the lumen interfaces. In order to optimize the image and reduce artefacts, the parameters were preset as follows: The highest frequency was set as $12 \mathrm{MHz}$; the lowest possible depth was set as $2.0 \mathrm{~cm}$ so that the artery of interest was at $\sim$ two thirsds of the display; the optimum dynamic range was set as 69 to increase the contrast of the image; and the operator adjusted the precise gain setting to 38 in order to visualize 2D images for the proximal and distal walls.

Following this, the hemodynamic assessment was conducted using the color flow mapping and pulse-Doppler technique. The color Doppler used the highest frequency as $7.5 \mathrm{MHz}$, the lowest possible pulse repetition frequency (PRF) of $1.4 \mathrm{kHz}$ to avoid aliasing, and the maximum color gain was set as 46 with minimum possible background noise. The pulse-Doppler examination used the highest frequency as $5.6 \mathrm{MHz}$ and the maximum power gain as 41. It was performed in the longitudinal view. Sample gate was placed within the lumen without touching the wall with an ultrasound incident angle $<60^{\circ}$.

Dissection and pathological histology. The patency of implanted grafts was monitored 4 and 8 weeks after implantation. Subsequently, the animals were sacrificed. The vascular grafts with native tissue segments above and below were explanted, rinsed with saline and fixed in a $4 \%$ paraformaldehyde solution overnight at $4^{\circ} \mathrm{C}$. The samples were embedded in paraffin, and then sectioned into $5-\mu \mathrm{m}$ sections. They were stained with hematoxylin for 8-10 min and then eosin for 30-60 sec at room temperature. Subsequently histological analysis was performed under a fluorescence microscope 

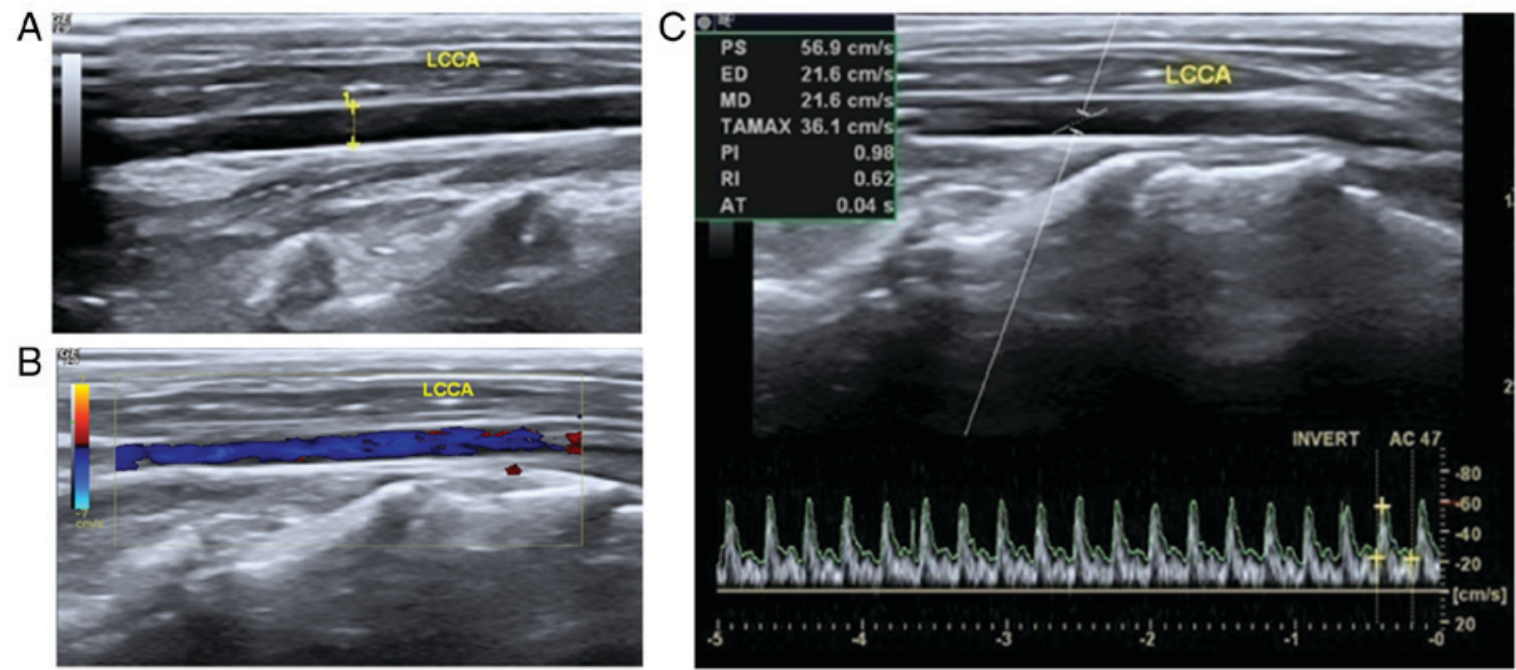

Figure 1. Long-axis section of the left common carotid artery as a control. (A) B-mode demonstrated normal anechoic lumen and hyperechoic linear wall. (B) Color flow mapping indicated the pure blue colorful filling of the vascular lumen. The blue color indicated the negative Doppler shifts and the direction of blood flow was receding from transducer, whereas the red color indicated positive Doppler shifts and the direction of blood flow was approaching from transducer. (C) Pulse-Doppler examination demonstrated a typical Doppler artery spectrum: Tall and sharp systolic peaks, and low and broad end-diastolic velocity. LCCA, left common carotid artery.

(Nikon Eclipse 80i; Nikon Corporation, Tokyo, Japan) at a magnification of $\times 5$.

Statistical analysis. PUSN was used as a control group and PUSN-Hep and PUSN-Hep/TPS were used as the experimental groups. Kaplan-Meier analysis was used to compare the patency rates of the groups and A log rank test also performed to compare the patency rates of the different groups by GraphPad Prism 5.0 software (GraphPad Software, Inc., La Jolla, CA, USA). A total of five repeats were performed. The data were analyzed by one-way analysis of variance. $\mathrm{P}<0.05$ was considered to indicate a statistically significant difference.

\section{Results}

Ultrasonography of the carotid artery without transplantation. As demonstrated in Fig. 1, clear images of the carotid arteries in the rabbits were generated by the use of transcutaneous ultrasound. The location and echo character, wall structure, as well as the caliber were bilaterally analyzed. As demonstrated in Fig. 1A, calipers and the dotted line indicated that the caliber was $0.2 \mathrm{~cm}$, and the carotid artery's blood flow pattern could be distinguished. Thus, the left-side artery without vascular prosthesis implantation was utilized as a control. Within a normal patent artery, a grayscale sonogram demonstrated the anechoic lumen and the hyperechoic linear wall of the vasculature (Fig. 1A). Color flow mapping indicated the pure color blood flow filling the vascular lumen (Fig. 1B). Furthermore, the regular arterial spectrum was detected based on pulse-Doppler sonogram examination. The pulsing patterns were tall and sharp systolic peaks with low and broad end diastolic velocity (Fig. 1C).

Distinguishing blood flow in grafts following transplantation in situ

Patency. Grayscale ultrasound demonstrated the clear anechoic within the graft of experimental group
PUSN-Hep/TPS, similar to the forward and the distal carotid vessels (Fig. 2A), which were filled with bright and pure blood flow signals during the color Doppler examination (Fig. 2B). In addition, sustained and steady artery blood flow spectrums were observed in the pulse-wave Doppler examination (Fig. 2C). The patency rate of all grafts was reflected clearly in the present study (Fig. 2D). PUSN grafts were all blocked $(0 / 5)$ at 4 weeks, while PUSN-Hep grafts demonstrated $80 \%$ patency $(4 / 5)$ at week 4 and $60 \%(3 / 5)$ at week 8 . The PUSN-Hep/TPS grafts indicated a patency of $60 \%(3 / 5)$ at 4 weeks, which was decreased to $20 \%(1 / 5)$ at 8 weeks. The differences between the control group (PUSN) and experimental groups (PUSN-Hep and PUSN-Hep/TPS) were statistically significant.

Stenosis. When the graft of control group PUSN was stenosed, heterogeneity was observed in iso- and hyper-echo within the lumen in grayscale sonography, albeit with varying anechoic areas (Fig. 3A). The forward and the distal carotid vessel cavity could be observed; however, their cavities were narrower than the normal carotid artery to varying degrees, as indicated in Fig. 3B. Calipers and a dotted line measured the caliber as $0.15 \mathrm{~cm}$, which was narrower than that of the control side (Fig. 1A). In addition, some hyperecho may have occurred in the anastomotic area (Fig. 3B). In the color Doppler map, some blood signals were observed within the graft; however, the blood signal could not be detected in the anechoic areas within the lumen (Fig. 3C). The pulse-Doppler sonogram examination revealed disorderly and unsystematic signals and noise, although some spectrums could still be obtained (Fig. 3D).

Occlusion. In grayscale sonography, heterogeneous hyperecho occurred within the lumen when the graft of control group PUSN was occluded. The forward and the distal carotid vessel cavity could not be clearly observed (Fig. 4A). In the color Doppler map, no blood flow signals were detected within the 

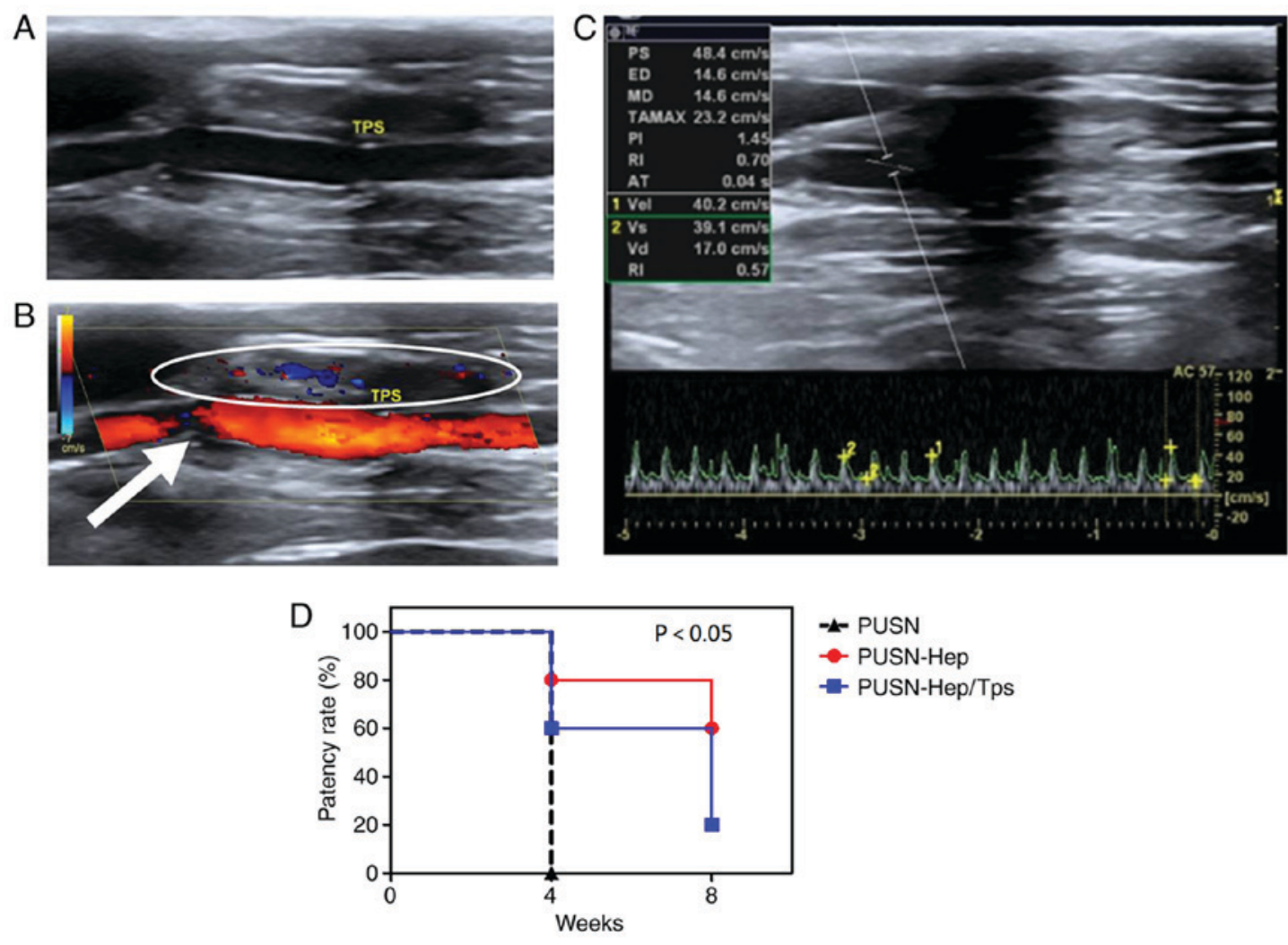

Figure 2. Long-axis section of the experimental group (PUSN-Hep/TPS) graft implanted after 4 weeks. (A) B-mode demonstrated a clear anechoic within the lumen, similar to the forward and the distal carotid vessels. (B) Color flow mapping indicated bright and pure red blood flow signals fulfilling the lumen of the forward and distal carotid vessels. There may have been a filling defect in the anastomotic (white arrow) due to the angulation; the circle indicates the background noise. (C) Pulse-Doppler examination demonstrated a typical artery spectrum with tall and sharp systolic peaks, and low and broad end-diastolic velocity was detected. (D) Patency rate of fibrous vascular grafts at weeks 4 and 8 after transplantation. $\mathrm{P}<0.05$ was considered to indicate a statistically significant difference between the PUSN group and PUSN-HEP and PUSN-HEP/Tps. PUSN, poly(ester urethane)urea with disulfide and amino groups; HEP, heparin; TPS, EPC recruiting peptide.
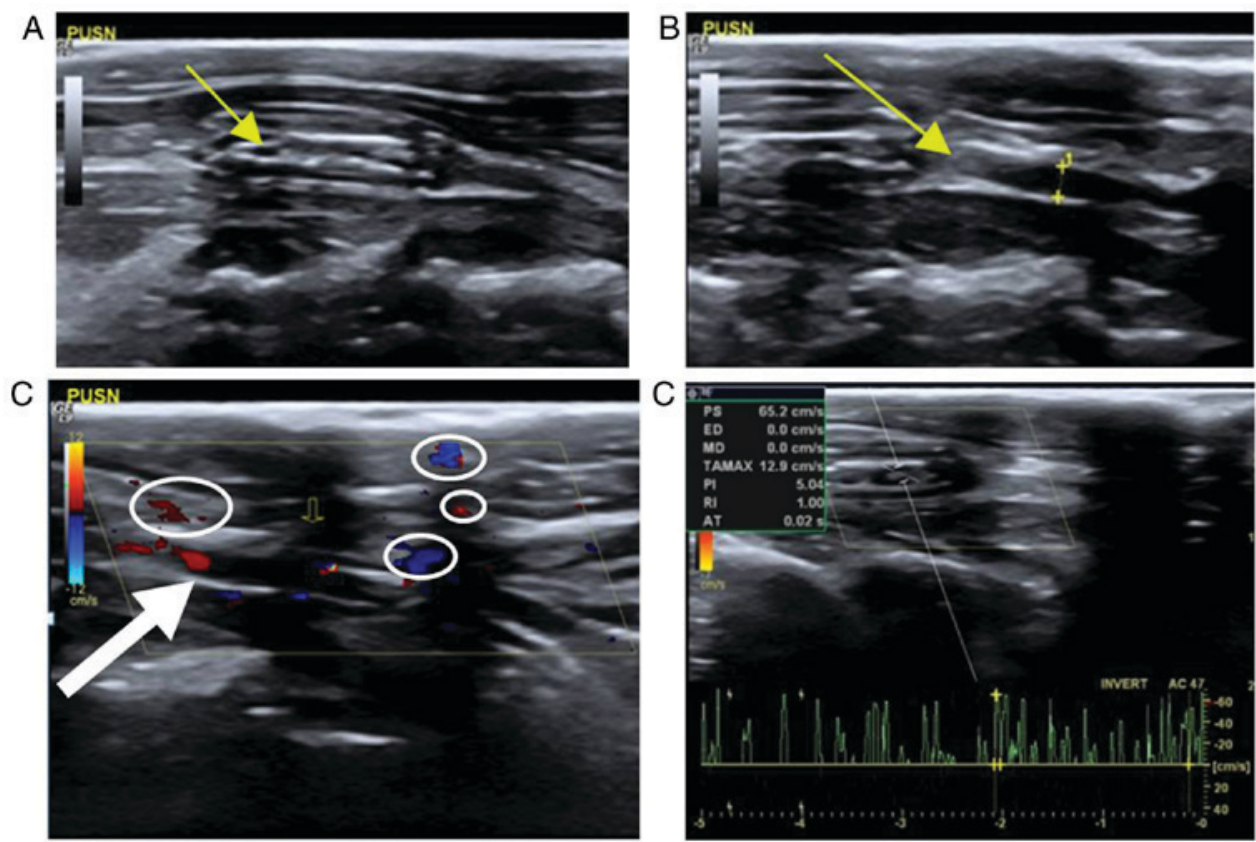

Figure 3. Long-axis section of the control (PUSN) group 4 weeks after graft implantation. (A) B-mode demonstrated heterogeneity in the iso- and hyperecho within the lumen. Arrow indicates small anechoic areas. (B) B-mode indicated that the distal carotid vessel could be observed with anechoic lumen. The arrow indicates some hyperechos in the anastomotic area. (C) The color flow map was tousled; there were some suspicious blood flow signals within the lumen (large white arrow), while in some anechoic areas of the lumen, blood flow signals could not be detected (small arrow). Circles indicate the surrounding background noise. (D) Pulse-Doppler examination demonstrated variable peaks with disorderly and unsystematic widths of the spectrum. PUSN, poly(ester urethane)urea with disulfide and amino groups. 

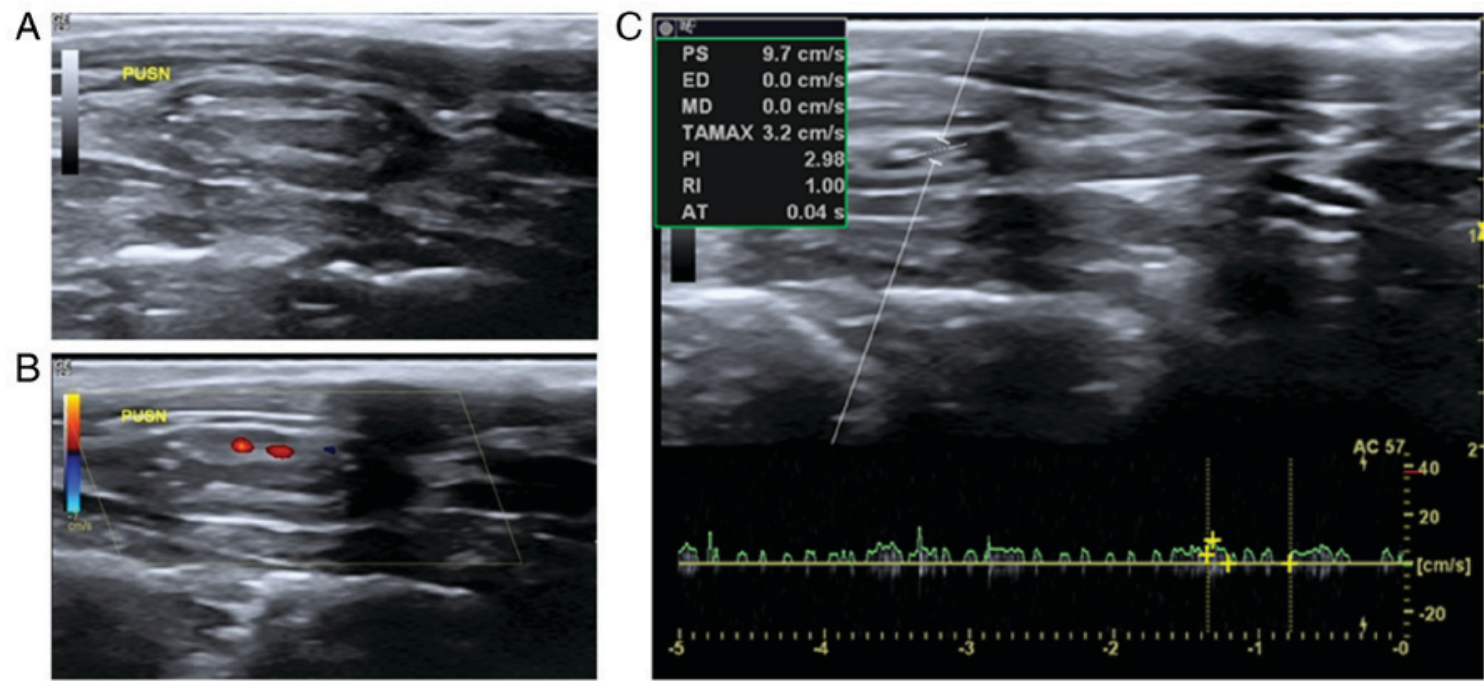

Figure 4. Long-axis section of the control (PUSN) group graft implanted after 8 weeks. (A) B-mode demonstrated heterogeneoushyperecho within the lumen, and the forward and distal carotid vessel cavities were not clearly visible. (B) Color flow mapping illustrated no blood signal within the lumen and few dotted color signals around the cavity, which were speculated as small lateral branches. (C) Pulse-Doppler examination demonstrated almost no arterial spectrums, only a few irregular wavelet noise spectrums with very low velocity were detected. PUSN, poly(ester urethane)urea with disulfide and amino groups.
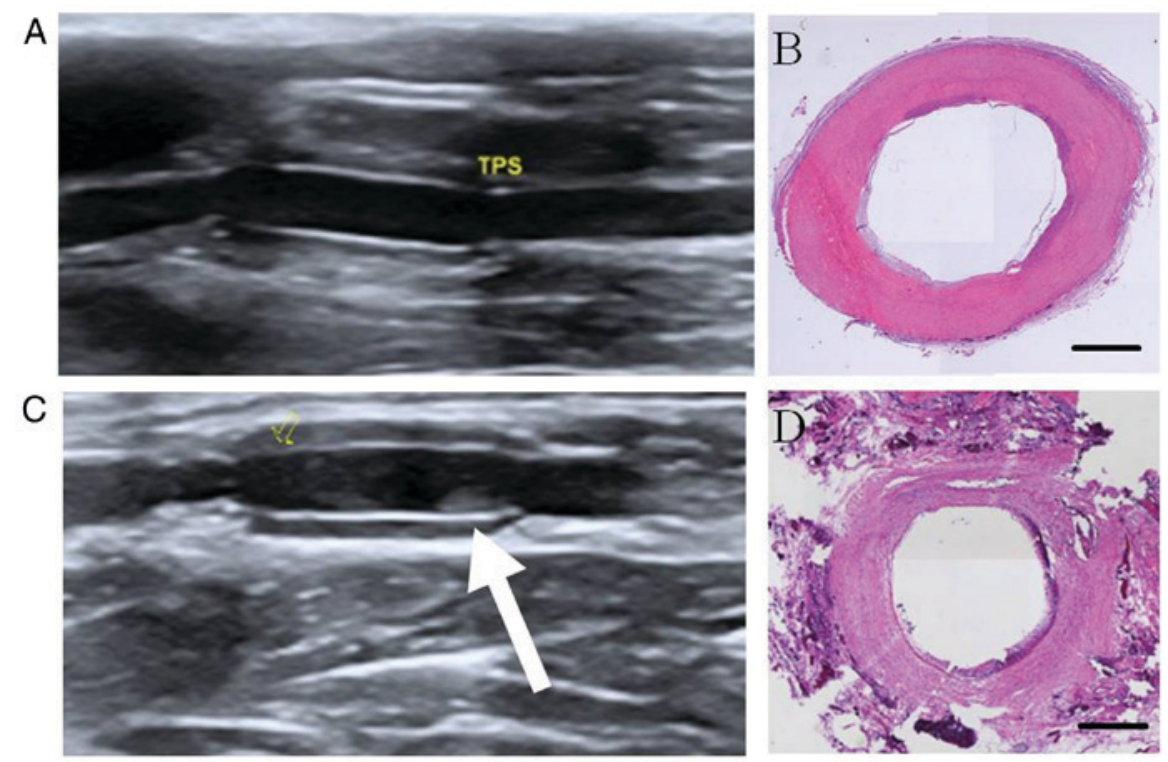

Figure 5. Experimental group grafts of PUSN-Hep/TPS and H\&E staining. (A) The experimental group grafts of PUSN-Hep/TPS 4 weeks after implantation. (B) H\&E staining of the fibrous graft in the experimental group after 4 weeks. (C) The experimental group grafts 8 weeks after implantation. Large white arrow indicates some iso-echo in the lumen, and the lucent pad of the lumen was less anechoic than in (A). The small yellow arrow indicates a narrower forward vessel than that observed in (A). (D) H\&E staining of the fibrous graft in the experimental group after 8 weeks. Scale bar, $1 \mathrm{~mm}$. H\&E, hematoxylin and eosin. TPS, EPC recruiting peptide.

lumen; however, a few dotted blood flow signals around the occluded cavity could be speculated as small lateral branches (Fig. 4B). Furthermore, few and irregular wavelet spectra, or even total lack of signals, were detected in the pulse-Doppler examination (Fig. 4C).

\section{Continuous monitoring of the flow changes in a graft}

Patency. For the patent vessels, there were few changes of echo charater within the lumen of grafts during the experimental period. After 4 weeks, a weak echo followed by iso-echo appeared gradually in the lumen of grafts, which indicated that their clear lumens gradually transformed to blurred lumens. Furthermore, as demonstrated in Fig. 5, the forward and/or the distal carotid vessels of experimental group PUSN-Hep/TPS became narrower over time (Fig. 5A and C). Fig. $5 \mathrm{~B}$ represents the matching histological staining image of Fig. 5A, demonstrating the smooth inner membrane, while Fig. 5D represents the matching histological staining image of Fig. 5C, displaying intimal hyperplasia in the lumen, corresponding to the stenosis trend detected by the ultrasound.

Severe stenosis. As demonstrated in Fig. 6, for the vessels of control group PUSN with severe stenosis, the heterogeneity 


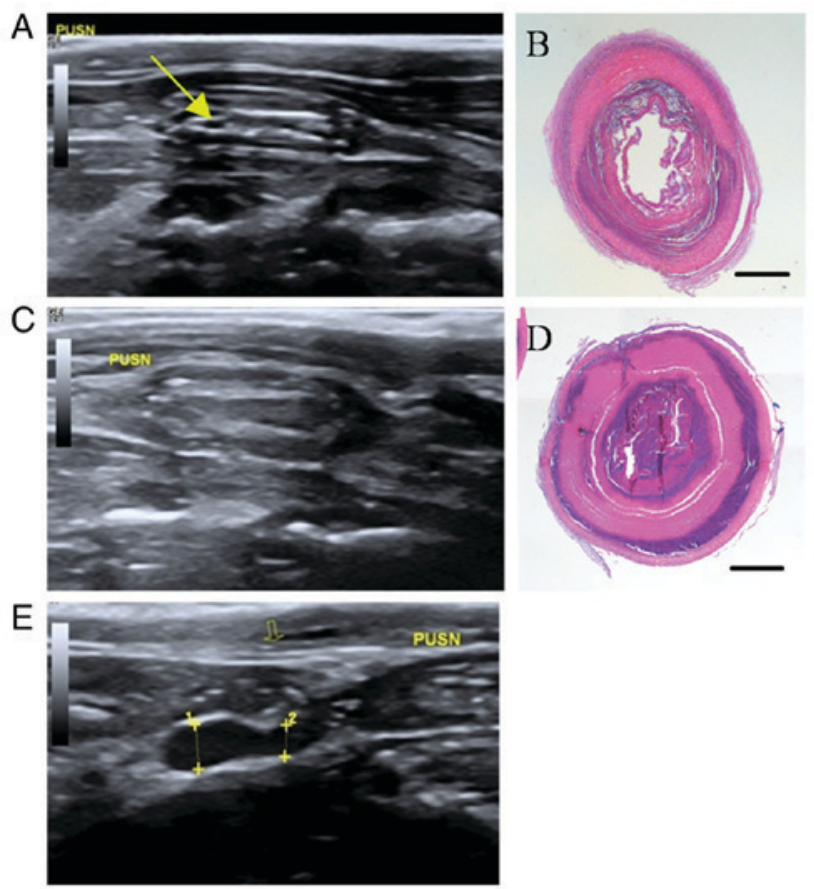

Figure 6. The control (PUSN) group grafts and their H\&E staining. (A) The control group graft 4 weeks after implantation. (B) H\&E staining of the control group fibrous graft after 4 weeks. (C) The control group graft 8 weeks after implantation. (D) H\&E staining of the control group fibrous graft after 8 weeks. (E) The forward blood vessel of the control group graft. Scale bar, $1 \mathrm{~mm}$. H\&E, hematoxylin and eosin. PUSN, poly(ester urethane)urea with disulfide and amino groups.

iso- and hyperecho of the graft (Fig. 6A) gradually transformed to homogeneous hyperecho (Fig. 6C). Notably, the narrow inner diameter of its forward vessel was measured by calipers and dotted line 2 as $0.16 \mathrm{~cm}$, and the expansion inner diameter of the distal stenosis was measured by calipers and dotted line 1 as $0.23 \mathrm{~cm}$ (Fig. 6E). Fig. 6B and D represent the matching histological staining images of Fig. 6A and $\mathrm{C}$, respectively, as substantiated by the stenosis condition of grafts that severely induced intraluminal thrombus and nearly resulted in total occlusion after 8 weeks of implantation.

Clear location of the grafts. High-frequency grayscale ultrasound has sufficient spatial resolution to distinguish the location of the graft conveniently on the surface. In B-mode sonography, it was possible to distinguish all of the anatomical layers of implanted grafts, including the lumen, graft wall, and the forward and distal carotid vessels (Fig. 2A). It was also possible to distinguish the location of anastomotic stenosis (Fig. 6E).

\section{Discussion}

Tissue engineering vascular grafts are a promising vascular substitute for the surgical treatment of diseased small-diameter vessels, particularly in young patients, with a wide prospect for clinical application (26). To assist in the clinical translation of tissue-engineered vascular grafts, angiography has been employed for assessing the patency of grafts (27). However, this invasive procedure is associated with the potential risks of vascular complications, including dissection, bleeding and pseudoaneurysm, and cannot monitor the longitudinal localization and function of grafts (28). The applicable non-invasive imaging modality is a superior choice in the tissue engineering experimental stage $(29,30)$. Vascular ultrasound techniques have been validated and are widely used in human subjects (31). Other imaging methods of carotid arteries may include digital subtraction angiography (DSA), MDCTA or MRA. DSA is considered the gold standard and is an indivisible part of the carotid stenting procedure; however, it is invasive (32). A study by Savic et al (14) demonstrated a significant agreement between MDCTA and ultrasound in the measurement of the degree and extent of carotid artery stenosis. However, the most notable disadvantages of MDCTA are the use of iodine contrast medium intravenously and radiation (32). MRA has refined the diagnostic capacity of non-invasive vascular imaging, and has progressively become competitive with DSA (15). MRA is more expensive than carotid US and computed tomography angiography (CTA) and is also less readily available. Furthermore, MRA is inferior in terms of spatial resolution compared with ultrasound and CTA due to its voxel view, and it may therefore not be possible to use it for direct visualization of small-diameter grafts (33). The general approach for diagnosis in patients with suspected carotid artery stenosis is to first perform US and then other non-invasive methods, including CTA and MRA $(14,32)$. Thus, the present research group aspire to introduce this useful tool in animal models under experimental conditions owing to its potential benefits.

Osorio-da Cruz et al (9) demonstrated that vascular ultrasound is a reliable non-invasive tool used for the routine assessment of vascular flow and patency in swine undergoing carotid graft replacement. However, no systematic study has revealed that vascular ultrasound may be used to monitor small-diameter $(<6 \mathrm{~mm})$ blood vessels, for example the carotid arteries of rabbits. The present study successfully imaged the carotid arteries of rabbits and implanted grafts for sufficient spatial resolution in order to monitor the patency and lumen characteristics of scaffolds.

In the present study, B-mode ultrasonography demonstrated excellent spatial resolution distinguishing the wall morphology and vascular composition, the lumen of grafts and their surrounding tissues. Also, it was possible to measure the diameter of the vessels for quantitative analysis of whether they were narrowing or expanding. For the clear double strong echo of the vascular graft wall, it was easy to identify the location of grafts, and hence, perform the body surface localization of the graft guided by vascular ultrasound. The experimental animals receiving graft implantation procedures require a structured surveillance strategy for follow-up care (34). The experimental rabbits in the present study exhibited an optimal wound healing ability. The surgical scars on their skin faded in $\sim 2$ weeks. After the skin scars faded, it was very difficult to label the exact location of implanted graft. The non-invasive skin localization may increase the probability of identification of an injury or severe inflammation (35). Specific types of radiolucent stents render difficulty in the localization of lost devices. Furthermore, the stent may be visualized by digital X-ray fluoroscopy; however, conventional fluoroscopy of the whole body involves considerable exposure to radiation designating 
the fluoroscopy as unrewarding (36). Presumably, it may be possible to attempt the localization of a misplaced stent with sonographic guidance in future clinical practice. We speculate that the non-invasive localization of grafts may be valuable in tissue engineering experiments, and subsequently clinical application.

Ultrasound is being used with increasing regularity in integrative human physiology research to determine the blood flow and changes in the blood vessel diameter in extracranial carotid arteries in response to experimental interventions (18). Thus, this imaging modality may also be used in laboratory animal studies to assess the physiological variables and hemodynamic changes of the stent-implanted arteries. The present study demonstrated that ultrasound has a high diagnostic accuracy in the characterization of graft stenosis or occlusions. The shift from B-mode image to velocity and hemodynamic parameters with increasing severity of an artery stent stenosis makes it possible to describe the entities with real physiological differences. The brighter the hue of red or blue, the higher the velocity of blood flow through the arteries (37). Based on the received Doppler frequency shift, spectral Doppler may be used to calculate the velocity of moving reflectors, such as red blood cells (38). In addition, the patency vessels with a vascular prosthesis may be analyzed quantitatively. When the vessels are obstructed, the spectrum curve becomes disorderly and unsystematic, and may not be detected.

In order to conduct the assessment, the animals should be placed in a supine position fixed on the examination couch with the neck fully exposed and hair shaved. Turning the head around $30^{\circ}$ towards the contralateral side elongates the neck sufficiently. However, applying excessive pressure on the neck with the transducer is avoided. Thus, the following criteria should be considered to acquire high-quality images. First, for the small-diameter arteries of rabbits, a linear transducer with high frequency should be used to increase the axial resolution. The parameters in B-mode should be preset as follows: Highest frequency; appropriate depth so that the artery of interest is at $\sim$ two thirds of the display; the focus should be adjusted to the point of interest; sufficient gain to clearly delineate the vessel wall from the lumen; decrease the dynamic range to increase the contrast of the image; and increase the filter noise rejection to eliminate the acoustic noise from weak echoes. Second, in the color or spectrum mode, the color box or the sample volume should be placed over the vessel of interest, the box steered to an adequate angle of $<60^{\circ}$ between the beam direction and the vessel, and the PRF should be set to detect the flow without spuriously superimposing color over tissue outside the vessel walls. The color gain is increased to satisfactory filling within the vessel without bleeding signals into the surrounding tissue. The procedure should be performed in multiple angles and directions in order to greatly avoid the interference signals. Finally, the ultrasound approach is a specialized technique, which is highly operator-dependent, requiring intensive training (18). Thus, it is recommended that the same operator performs the procedure during a lab process for consistent and reliable results.

In the present study, ultrasound was beneficial to discriminate near-occlusion from occlusion of the carotid grafts in rabbits due to the visualization of the residual lumen on the
B-mode image or little blood flow signals on color Doppler. Presently, the ultrasound B-mode is the most effective method for demonstrating minor arterial plaques, although color flow imaging is vital for velocity measurement and essential for differentiating occlusion from stenosis (39). The grayscale ultrasonography may present a higher spatial resolution as compared to color and power Doppler examination; the level of purity of the graft lumen may serve as a considerable parameter to judge its patency. During mild-to-severe stenosis, and also occlusion, gradually increased iso- to hyperecho flocculent masses occurred in the graft lumens, considering the amount of platelet deposition for the turbulent blood flow. Furthermore, there may be some blood flow signals in the lumens, which are 'mosaic' colored for those that are not completely occluded. During total occlusion of the lumens, few dotted blood flow signals around the cavity were observed and it was speculated that tiny collateral vessels formatted around the occluded vascular graft for compensation. Owing to the non-invasive, fast, non-ionizing and cost-effective modality, the present research group recommends vascular Doppler ultrasonography as the superior choice for continuous surveillance of vascular grafts with almost no contraindications. Additionally, it may repeatedly be used without animal sacrifice, to provide valuable information about the grafts, thereby greatly shortening the experimental period.

The most notable limitation of the present study was that the measurement of the diameter in different blood flow situations in vivo was not quantified, which suggests that further study is required. There were also some background noises in the color map, which may have disturbed the evaluation of the blood flow pattern to some degree. Doppler color flow image quality is controlled by multi-factor parameter settings, including gain, time gain control, ensemble length, Nyquist limit (PRF), baseline shift, wall filter, color window angle, location and size (40). The check and balance of these complicated and numerous parameter settings will generate some background noise, which was also a limitation of the present study. Further work should be performed in the selection of the appropriate instrument models and the most effective combination of parameter settings.

\section{Acknowledgements}

The authors would like to thank Mr. Jun Fang, an the important member in our synthetic vascular graft experimental group of Shanghai Children's Medical Center, for his constant encouragement and guidance.

\section{Funding}

The present study was supported by the National Natural Science Foundation of China (grant no. 81671833) and the Collaborative Innovation Center for Translational Medicine at Shanghai Jiao Tong University School of Medicine (grant no. TM201504).

\section{Availability of data and materials}

All data generated or analyzed during this study are included in this published article. 


\section{Authors' contributions}

JS, JLZ and JD designed the study. JS, JLZ and JD performed the experiments, while QW and MY analyzed data. JS and JLZ wrote the manuscript, MY assisted in preparing the manuscript. JD and QW reviewed and edited the manuscript. All authors read and approved the final manuscript.

\section{Ethics approval and consent to participate}

All experimental protocols were approved by the Animal Care and Experiment Committee of Shanghai Jiao Tong University School of Medicine (Shanghai, China).

\section{Consent for publication}

Not applicable.

\section{Competing interests}

The authors declare that they have no competing interests.

\section{References}

1. Dahl SL, Kypson AP, Lawson JH, Blum JL, Strader JT, Li Y, Manson RJ, Tente WE, DiBernardo L, Hensley MT, et al: Readily available tissue-engineered vascular grafts. Sci Transl Med 68: 68ra9, 2011.

2. Wu T, Zhang J, Wang Y, Li D, Sun B, El-Hamshary H, Yin M and Mo X: Fabrication and preliminary study of a biomimetic tri-layer tubular graft based on fibers and fiber yarns for vascular tissue engineering. Mater Sci Eng C Mater Biol Appl 82: 121-129, 2018.

3. Zhang J, Du J, Xia D, Liu J, Wu T, Shi J, Song W, Jin D, Mo X and Yin M: Preliminary study of a novel nanofiber-based valve integrated tubular graft as an alternative for a pulmonary valved artery. RSC Adv 6: 84837-84846, 2016.

4. Zilla P, Bezuidenhout D and Human P: Prosthetic vascular grafts: Wrong models, wrong questions and no healing. Biomaterials 34 5009-5027, 2007.

5. Thomas LV, Lekshmi V and Nair PD: Tissue engineered vascular grafts-preclinical aspects. Int J Cardiol 167: 1091-1100, 2013.

6. Nagaoka Y, Yamada H, Kimura T, Kishida A, Fujisato T and Takakuda K: Reconstruction of small diameter arteries using decellularized vascular scaffolds. J Med Dent Sci 61: 33-40, 2014.

7. Kurobe H, Maxfield MW, Tara S, Rocco KA, Bagi PS, Yi T, Udelsman B, Zhuang ZW, Cleary M, Iwakiri Y, et al: Development of small diameter nanofiber tissue engineered arterial grafts. PLoS One 10: e0120328, 2015.

8. Itoda Y, Panthee N, Tanaka T, Ando T, Sakuma I and Ono M: Novel anastomotic device for distal coronary anastomosis: Preclinical results from swine off-pump coronary artery bypass model. Ann Thorac Surg 101: 736-741, 2016.

9. Osorio-da Cruz SM, Aggoun Y, Cikirikcioglu M, Khabiri E, Djebaili K, Kalangos A and Walpoth B: Vascular ultrasound studies for the non-invasive assessment of vascular flow and patency in experimental surgery in the pig. Lab Anim 43: 333-337, 2009.

10. Culp BC, Brown AT, Erdem E, Lowery J and Culp WC: Selective intracranial magnification angiography of the rabbit: Basic techniques and anatomy. J Vasc Interv Radiol 18: 187-192, 2007.

11. Fujii T, Fukuyama N, Tanaka C, Ikeya Y, Shinozaki Y, Kawai T, Atsumi T, Shiraishi T, Sato E, Kuroda R, et al: Visualization of microvessels by angiography using inverse-Compton scattering X-rays in animal models. J Synchrotron Radiat 21: 1327-1332, 2014

12. Towner RA, Smith N, Asano Y, He T, Doblas S, Saunders D, Silasi-Mansat R, Lupu F and Seeney CE: Molecular magnetic resonance imaging approaches used to aid in the understanding of angiogenesis in vivo: Implications for tissue engineering. Tissue Eng Part A 16: 357-364, 2010.
13. Liu H, Wang X, Tan KB, Liu P, Zhuo ZX, Liu Z, Hua X, Zhuo QQ, Xia HM and Gao YH: Molecular imaging of vulnerable plaques in rabbits using contrast-enhanced ultrasound targeting to vascular endothelial growth factor receptor-2. J Clin Ultrasound 39: 83-90, 2011.

14. Savic ZN, Davidovic LB, Sagic DZ, Brajovic MD and Popovic SS: Correlation of color Doppler with multidetector CT angiography findings in carotid artery stenosis. ScientificWorldJournal 10: 1818-1825, 2010

15. D'Onofrio M, Mansueto G, Faccioli N, Guarise A, Tamellini P Bogina G and Pozzi Mucelli R: Doppler ultrasound and contrast-enhanced magnetic resonance angiography in assessing carotid artery stenosis. Radiol Med 111: 93-103, 2006 (In English, Italian).

16. Grant EG, Benson CB, Moneta GL, Alexandrov AV, Baker JD, Bluth EI, Carroll BA, Eliasziw M, Gocke J, Hertzberg BS, et al: Carotid artery stenosis: Grayscale and Doppler ultrasound diagnosis-Society of Radiologists in Ultrasound consensus conference. Ultrasound Q 19: 190-198, 2003.

17. Crişan S: Carotid ultrasound. Med Ultrason 13: 326-330, 2011.

18. Thomas KN, Lewis NC, Hill BG and Ainslie PN: Technical recommendations for the use of carotid duplex ultrasound for the assessment of extracranial blood flow. Am J Physiol Regul Integr Comp Physiol 309: R707-R720, 2015.

19. Högberg D, Dellagrammaticas D, Kragsterman B, Björck M and Wanhainen A: Simplified ultrasound protocol for the exclusion of clinically significant carotid artery stenosis. Ups J Med Sci 121: 165-169, 2016

20. Fang J, Zhang J, Du J, Pan Y, Shi J, Peng Y, Chen W, Yuan L, Ye SH, Wagner WR, et al: Orthogonally functionalizable polyurethane with subsequent modification with heparin and endothelium-inducing peptide aiming for vascular reconstruction. ACS Appl Mater Interfaces 8: 14442-14452, 2016.

21. Shastri VP: In vivo engineering of tissues: Biological considerations, challenges, strategies, and future directions. Adv Mater 21: 3246-3254, 2009.

22. Hollister SJ: Scaffold design and manufacturing: From concept to clinic. Adv Mater 21: 3330-3342, 2009.

23. Pashuck ET and Stevens MM: Designing regenerative biomaterial therapies for the clinic. Sci Transl Med 4: 160sr4, 2012.

24. Appel AA, Anastasio MA, Larson JC and Brey EM: Imaging challenges in biomaterials and tissue engineering. Biomaterials 34: 6615-6630, 2013.

25. Zheng W, Wang Z, Song L, Zhao Q, Zhang J, Li D, Wang S, Han J, Zheng XL, Yang Z and Kong D: Endothelialization and patency of RGD-functionalized vascular grafts in a rabbit carotid artery model. Biomaterials 33: 2880-2891, 2012.

26. Benrashid E, McCoy CC, Youngwirth LM, Kim J, Manson RJ, Otto JC and Lawson JH: Tissue engineered vascular grafts: Origins, development, and current strategies for clinical application. Methods 99: 13-19, 2016.

27. Chan M, Ridley L, Dunn DJ, Tian DH, Liou K, Ozdirik J, Cheruvu $\mathrm{C}$ and Cao C: A systematic review and meta-analysis of multidetector computed tomography in the assessment of coronary artery bypass grafts. Int J Cardiol 221: 898-905, 2016.

28. Levine GN, Bates ER, Blankenship JC, Bailey SR, Bittl JA, Cercek B, Chambers CE, Ellis SG, Guyton RA, Hollenberg SM, et al: 2011 ACCF/AHA/SCAI guideline for percutaneous coronary intervention: A report of the American College of cardiology foundation/American heart association task force on practice guidelines and the society for cardiovascular angiography and interventions. Circulation 124: e574-e651, 2011.

29. Hjortnaes J, Gottlieb D, Figueiredo JL, Melero-Martin J, Kohler RH, Bischoff J, Weissleder R, Mayer JE and Aikawa E: Intravital molecular imaging of small-diameter tissue-engineered vascular grafts in mice: A feasibility study. Tissue Eng Part C Methods 16: 597-607, 2010.

30. Mertens ME, Koch S, Schuster P, Wehner J, Wu Z, Gremse F, Schulz V, Rongen L, Wolf F, Frese J, et al: USPIO-labeled textile materials for non-invasive MR imaging of tissue-engineered vascular grafts. Biomaterials 39: 155-163, 2015

31. Rübenthaler J, Reiser $M$ and Clevert DA: Diagnostic vascular ultrasonography with the help of color Doppler and contrast-enhanced ultrasonography. Ultrasonography 35: 289-301, 2016.

32. Adla T and Adlova R: Multimodality imaging of carotid stenosis. Int J Angiol 24: 179-184, 2015. 
33. Jaff MR, Goldmakher GV, Lev MH and Romero JM: Imaging of the carotid arteries: The role of duplex ultrasonography, magnetic resonance arteriography, and computerized tomographic arteriography. Vasc Med 13: 281-292, 2008.

34. Thukkani AK and Kinlay S: Endovascular intervention for peripheral artery disease. Circ Res 116: 1599-1613, 2015.

35. Berland TL, Smith SL, Metzger PP, Nelson KL, Fakhre GP, Chua HK, Burnett OL, Falkensammer J, Hickman HJ and Hinder RA: Intraoperative gamma probe localization of the ureters: A novel concept. J Am Coll Surg 205: 608-611, 2007.

36. Mohiaddin RH, Roberts RH, Underwood R and Rothman M: Localization of a misplaced coronary artery stent by magnetic resonance imaging. Clin Cardiol 18: 175-177, 1995.

37. Merritt CR: Doppler color imaging. Introduction. Clin Diagn Ultrasound 27: 1-6, 1992.
38. Browne JE: A review of Doppler ultrasound quality assurance protocols and test devices. Phys Med 30: 742-751, 2014.

39. von Reutern GM, Goertler MW, Bornstein NM, Del Sette M, Evans DH, Hetzel A, Kaps M, Perren F, Razumovky A, von Reutern M, et al: Grading carotid stenosis using ultrasonic methods. Stroke 43: 916-921, 2012.

40. Kremkau FW: Doppler color imaging. Principles and instrumentation. Clin Diagn Ultrasound 27: 7-60, 1992.

(i) (2) This work is licensed under a Creative Commons

EY No ND Attribution-NonCommercial-NoDerivatives 4.0 International (CC BY-NC-ND 4.0) License. 